Meta

Journal des traducteurs

Translators' Journal

\title{
Colloque sur l'enseignement de la traduction juridique
}

\section{Geneviève Mareschal}

Volume 30, numéro 4, décembre 1985

URI : https://id.erudit.org/iderudit/003571ar

DOI : https://doi.org/10.7202/003571ar

Aller au sommaire du numéro

Éditeur(s)

Les Presses de l'Université de Montréal

ISSN

0026-0452 (imprimé)

1492-1421 (numérique)

Découvrir la revue

Citer cette note

Mareschal, G. (1985). Colloque sur l'enseignement de la traduction juridique. Meta, 30(4), 407-407. https://doi.org/10.7202/003571ar d'utilisation que vous pouvez consulter en ligne.

https://apropos.erudit.org/fr/usagers/politique-dutilisation/ 


\section{COLLOQUE SUR L'ENSEIGNEMENT DE LA TRADUCTION JURIDIQUE}

Les 21 et 22 février 1985, l'École de traducteurs et d'interprètes de l'Université d'Ottawa organisait un colloque sur l'enseignement de la traduction juridique. Ce colloque réunissait des employeurs de traducteurs juridiques et des enseignants et avait pour but de soumettre à la critique du milieu professionnel et universitaire le programme spécialisé de traduction juridique que l'École prévoit lancer en septembre 1986 .

Le programme du colloque avait été conçu de façon à mettre d'abord l'accent sur la situation de la traduction juridique et du traducteur juridique au Canada, puis de décrire la situation idéale souhaitée par les employeurs et, enfin, de voir dans quelle mesure le nouveau programme de traduction juridique permettrait de combler le fossé séparant l'idéal de la réalité.

La première moitié du colloque a permis aux participants de faire le point sur la question. Les employeurs de traducteurs juridiques des secteurs public et privé ont décrit, chacun à leur tour, les services de traduction qu'ils dirigent, leur mode de fonctionnement, les compétences juridiques et linguistiques de leurs traducteurs et réviseurs, les problèmes de recrutement auxquels ils ont à faire face, les points forts et les points faibles de leur organisation et ont été amenés, en conclusion, à exprimer leurs véritables attentes quant à la formation professionnelle des candidats qu'ils ont à engager. Le monde professionnel a ensuite cédé la parole au monde universitaire, et les titulaires des cours de traduction juridique des différents programmes universitaires de traduction ont entrepris d'exposer le contenu de leurs cours, de préciser la place accordée à celui-ci dans leurs programmes respectifs et d'en évaluer l'efficacité et l'intérêt.

Des propos tenus par les employeurs et les enseignants, il est vite ressorti que la formation actuelle des traducteurs juridiques comportait bien des lacunes. L'annonce de la mise sur pied d'un programme universitaire, consacré spécifiquement à l'enseignement de la traduction juridique, a donc reçu un accueil positif. C'est à la présentation et à l'étude de ce programme qu'a été consacrée la deuxième journée du colloque. Le programme de traduction juridique est un programme de deuxième cycle, qui exige une formation première soit en traduction, soit en droit. Après une série de cours visant à former les candidats dans la discipline complémentaire (droit pour les traducteurs, traduction pour les juristes), les deux catégories d'étudiants poursuivent ensemble l'apprentissage de traduction juridique proprement dite. Le programme se termine par un stage obligatoire dans un cabinet ou service de traduction juridique. Les débats et discussions auxquels a donné lieu l'analyse du nouveau programme ont permis de définir plus précisément la complexité de la situation juridique au Canada, la diversité et l'étendue des compétences et des connaissances à acquérir et la clientèle visée par un programme de ce genre. Ils ont aussi suscité quelques suggestions originales et fort intéressantes quant au contenu du programme proposé. Un compte rendu détaillé du colloque a été publié dans la collection « Documents de traductologie " de l'École de traducteurs et d'interprètes et est diffusé par les Presses de l'Université d'Ottawa.

GENEVIÈvE MARESCHAL 